\title{
Charged donors in quantum dots: Finite difference and fractional dimensions results
}

\author{
C. Riva, ${ }^{1, *}$ R. A. Escorcia, ${ }^{1,2}$ A. O. Govorov, ${ }^{3}$ and F. M. Peeters ${ }^{1, \dagger}$ \\ ${ }^{1}$ Departement Natuurkunde, Universiteit Antwerpen (Campus Drie Eiken), Universiteitsplein 1, B-2610 Antwerpen, Belgium \\ ${ }^{2}$ Facultad de Ciencias Básicas, Universidad del Magdalena, A. A. 731 Santa Marta, Colombia \\ ${ }^{3}$ Department of Physics and Astronomy, Ohio University, Athens, Ohio 45701, USA
}

(Received 3 November 2003; published 14 June 2004)

\begin{abstract}
Neutral and charged shallow donor states confined by a parabolic potential in the plane of a GaAs quantum well are studied in the presence of a perpendicular magnetic field. The influence of the position of the donor on the energy of the states is studied, i.e., we consider both the cases when the donor impurity is on and off the center of the dot. We investigate the dependence of the ground and excited states on the confinement potential and on the presence of an external magnetic field. Two different theoretical approaches are used: the finite difference technique and the fractional-dimension method. We found that if the donor is displaced from the center of the quantum well, the presence of the lateral confinement shifts the magnetic field-induced angular momentum transitions and the unbinding of the charged donor state to a lower magnetic field.
\end{abstract}

DOI: 10.1103/PhysRevB.69.245306 PACS number(s): 73.21.La, 73.21.Fg, 71.55.-i, 78.67.De

\section{INTRODUCTION}

In the past decade, there has been increasing interest in the properties of negatively charged donors $\left(D^{-}\right.$centers) in quasi-two-dimensional (2D) systems, particularly in quantum wells $(\mathrm{QWs})^{1-13}$ and in quantum dots (QDs). ${ }^{14,15}$ The $D^{-}$ centers can be formed in bulk semiconductors only under metastable conditions, ${ }^{2}$ however, in semiconductor heterostructures these are readily formed because of the dramatic increase of the $D^{-}$binding energy as a consequence of the spatial confinement, which is even further increased by the application of a magnetic field. Different theoretical approaches have been used to calculate the spectra of $D^{-}$systems in QWs and QDs, such as the diffusion quantum Monte Carlo method, ${ }^{3,4}$ the finite difference technique, ${ }^{12}$ and the variational method. ${ }^{8,9}$ Recently, $D^{-}$systems have been studied in quantum dots. In particular, the problem of the neutral and charged donor in a parabolic $2 \mathrm{D}$ quantum dot were studied, ${ }^{14,15}$ both in the case when the donor is lying in the confinement plane and when it is displaced outside the plane.

In spite of all the different approaches used, it is known that the best variational results for the ground-state energy of a $D^{-}$ion in three- and exact two-dimensional semiconductors were obtained by using a Hylleraas trial function with 35 linear variational parameters. ${ }^{1}$ For the $D^{-}$system in semiconductor heterostructures the advantages of this trial function vanish due to the anisotropy imposed by the confinement potential and the external field, however, this approach seems still possible if one reduces the nanostructure problem to an isotropic one in a space with noninteger dimensions. ${ }^{13}$ Such an approach was previously applied to the neutral $\left(D^{0}\right)$ and charged $\left(D^{-}\right)$systems in a QW. ${ }^{19,20}$

The method of fractional-dimensional space was used to study shallow donor impurities and excitons in quantumconfined semiconductor structures. ${ }^{16-18}$ In this approach the anisotropic problem in a three-dimensional environment is mapped to an isotropic one in a lower effective fractionaldimensional space, where the dimension is related to the anisotropy introduced both by the heterostructure barrier poten- tial and the magnetic field. The method used in Ref. 16 is rather simple but the problem is that it is not clear how to map a real problem with confinement to a problem with fractional dimensions where the confinement is no longer present. As a consequence, this elegant method is not relevant for practical systems. Recently, a new method was introduced in the study of the ground state of neutral $\left(D^{0}\right)$ and charged donors $\left(D^{-}\right)$in QWs and QDs. ${ }^{19,20}$ In this procedure, the $D^{0}$ and $D^{-}$systems in semiconductor heterostructures were reduced to similar ones in an isotropic effective space with a nonfixed fractional dimension. Subsequently, a threeparameter Hylleraas trial function was used to solve the renormalized Schrödinger equation for the $D^{-}$system. The method provides a simple and unified procedure for calculating the $D^{0}$ and $D^{-}$ground-state binding energies in different heterostructures with arbitrary confining potential. Its accuracy has been tested by comparison with theoretical calculations from Monte Carlo and variational methods using Chandrasekhar-type trial functions.

In this paper, we study the ground and first excited state of a $D^{-}$in a quantum dot of finite thickness $W$, which we model as a $\mathrm{QW}$ in the $z$ direction and a parabolic potential of frequency $\omega_{0}$ in the $x y$ plane. In Sec. II we present the model and discuss how the finite difference method (FDiff) and the fractional-dimension method (FDim) are used to solve the problem. In Sec. III we compare and discuss the results obtained with the two techniques. In Sec. IV we present and discuss our results for the charged donor in a quantum dot as function of the donor position, the confinement potential, and the presence of an external magnetic field. In Sec. V we summarize our results and conclusions.

\section{THE MODEL AND THE APPROXIMATIONS}

We consider a $D^{-}$center in a quantum dot in the presence of an external magnetic field. The QD is modeled as a QW with square-well confinement potential and width $W$ in the $z$ direction, and parabolic potential with frequency $\omega_{0}$ in the $x y$ plane. Such a situation is present in many quantum wells 
where well width fluctuations lead to an additional lateral confinement of the particles which we model by a simple parabolic potential. Alternatively, such parabolic in-plane confinement can be realized, e.g., by using a set of gates on top of the QW. We use the effective Bohr radius $a_{B}$ $=\varepsilon \hbar^{2} / m^{*} e^{2}$, the effective Rydberg $R_{y}=e^{2} / 2 \varepsilon a_{B}$, and $\gamma_{c}$ $=e \hbar B / 2 m^{*} R_{y}$ as units of length, energy, and magnetic field, respectively. We will apply our results to the case of a $\mathrm{GaAs} / \mathrm{AlGaAs}$ nanostructure where the respective values are $R_{y}=5.8 \mathrm{meV}, a_{B}=99.7 \AA$ and $\gamma_{c}=0.148 B(T)$. Within the framework of the effective-mass approximation and assuming the Coulomb gauge for the magnetic field, i.e., $\mathbf{A}=\mathbf{r}$ $\times \mathbf{B} / 2$, the dimensionless Hamiltonian for the $D^{-}$center in the QD can be written in cylindrical coordinates as

$$
\begin{gathered}
H=H_{0}\left(\mathbf{r}_{1}\right)+H_{0}\left(\mathbf{r}_{2}\right)+\frac{2}{r_{12}}, \\
H_{0}\left(\mathbf{r}_{i}\right)=-\nabla_{i}^{2}+V_{i}\left(z_{i}\right)+\frac{1}{4}\left(\omega^{2}+\gamma_{c}^{2}\right) \rho_{i}^{2}-i \gamma_{c} \frac{\partial}{\partial \varphi}-\frac{2}{\left|\mathbf{r}_{i}-\zeta\right|},
\end{gathered}
$$

where $\zeta=(0,0, \zeta)$ is the position of the impurity ion with respect to the center of the well, $\mathbf{r}_{\mathbf{i}}$ is the $i$ th electron position vectors, $r_{12}=\left|\mathbf{r}_{1}-\mathbf{r}_{2}\right|$ is the electron-electron distance, $V_{i}\left(z_{i}\right)$ is the confinement potential, which is zero in the quantum well, and $V_{e}=0.6 \times\left(1.155 x+0.37 x^{2}\right) \mathrm{eV}$ outside the well, with $x=0.3$ in our case being the concentration of $\mathrm{Al}$ in the GaAsAl barrier; $\omega=\hbar \omega_{0} / 2 R_{y}$ with $\omega_{0}$ is the confining frequency corresponding to the in-plane parabolic potential $V(\rho)=\omega_{0}^{2} \rho^{2} / 2$.

\section{A. Finite difference technique (FDiff)}

In the finite difference approach we follow a quasiadiabatic approach. We write the wave function for the $D^{-}$as

$$
\psi(\mathbf{r})=f_{0}\left(z_{1}\right) f_{0}\left(z_{2}\right) \psi\left(\rho_{1}, \rho_{2}\right),
$$

where $f_{0}\left(z_{i}\right)$ is the solution for an electron in a $\mathrm{QW}$ of width equal to the thickness of the quantum dot and $\psi\left(\rho_{1}, \rho_{2}\right)$ is obtained as a solution of the Hamiltonian,

$$
H=H_{0}\left(\mathbf{r}_{1}\right)+H_{0}\left(\mathbf{r}_{2}\right)+V_{e f f}\left(\rho_{12}\right),
$$

where

$$
H_{0}(\mathbf{r})=-\nabla_{i}^{2}+\frac{1}{4}\left(\omega^{2}+\gamma_{c}^{2}\right) \rho_{i}^{2}-i \gamma_{c} \frac{\partial}{\partial \varphi}-V_{e f f}\left(\rho_{i}\right),
$$

and $V_{\text {eff }}(\rho)$ is obtained averaging the Coulomb interaction in the $z$ direction over the $z$-confinement wave functions $f_{0}\left(z_{1}\right) f_{0}\left(z_{2}\right)$. The resulting Schrödinger equation for the neutral donor is solved exactly, using the finite difference technique. In a second step the Schrödinger equation for the charged donor is solved using as a wave function a linear combination of neutral donor functions and angular harmonics. The approximation and the approach followed are explained in depth in Refs. 12 and 21. This approximation is justified when the potential in the $z$ direction is strong compared to the Coulomb interaction. This technique was al- ready used successfully to study the off-center donor in a QW, see Ref. 12.

\section{B. Fractional-dimension method (FDim)}

In the fractional-dimension method we first solve the problem of the free electron in a $\mathrm{QW}$ in the presence of a magnetic field which can be written as

$$
H_{e}(\mathbf{r}) f_{n, m}(\mathbf{r})=E_{n, m} f_{n, m}(\mathbf{r}),
$$

with

$$
H_{e}(\mathbf{r})=-\nabla_{\rho, i}^{2}+V\left(z_{i}\right)+\frac{1}{4}\left(\omega^{2}+\gamma^{2}\right) \rho_{i}^{2}-i \gamma \frac{\partial}{\partial \varphi}
$$

and

$$
f_{0, m}(\mathbf{r})=f_{0}(z) R_{m}(\rho) \exp (i m \varphi),
$$

where $f_{0}(z)$ is the lowest energy solution for the free electron in a QW without a magnetic field, with the energy $E_{z}$, and where $R_{m}(\rho)$ is the radial part of the wave function for the circular oscillator in the presence of a magnetic field: ${ }^{22}$

$$
\begin{aligned}
R_{n_{r}, m}(\rho)= & \rho^{|m|} \exp \left(-\gamma \rho^{2} / 4\right)_{1} F_{1}\left(-n_{r},|m|+1 ; \gamma \rho^{2}\right) ; \\
& \gamma^{2}=\gamma_{c}^{2}+\omega^{2} .
\end{aligned}
$$

Here ${ }_{1} F_{1}$ is the confluent hypergeometric function, $n_{r}$ is the radial quantum number, and $m$ is the magnetic quantum number. The corresponding energy levels are then given by

$$
\begin{aligned}
E_{0, n_{r}, m}= & E_{0, z}-\gamma_{c} m+\gamma\left(|m|+1+2 n_{r}\right), \\
& m=0, \pm 1, \pm 2, \ldots, \quad n_{r}=0,1,2 \ldots,
\end{aligned}
$$

and the wave function for the ground and the first excited state are

$$
f_{0, m}(\mathbf{r})=f_{0}(z) \rho^{|m|} \exp \left(-\gamma \rho^{2} / 4\right) \exp (i m \varphi), \quad m=0, \pm 1 .
$$

From now on we will no longer write the subscript 0 , which indicates that for the electron in the $z$ direction we consider only the lowest QW level, as this is true through the whole paper. In order to solve the problem for a $D^{0}$ impurity in a $\mathrm{QD}$, we choose the following trial wave function, which takes into account the possible asymmetry of the system along the $z$ axis produced by the displacement of the impurity:

$$
\Psi_{D^{0}}^{(m)}(\mathbf{r})=f_{m}(\mathbf{r}) \phi_{D^{0}}^{(m)}(|\mathbf{r}-\zeta|) .
$$

Here $f_{m}(\mathbf{r})$ is the wave function for a free electron in the $m$ th state given by Eq. (8) and $\phi_{D^{0}}^{(m)}$ is the envelope wave function which depends on the distance of the electron from the origin of the coordinate system located at the donor position. As explained in Refs. 19 and 20 we start from the Schrödinger variational principle to formulate the corresponding problem for $D^{0}$ in a $\mathrm{QW}$ as a variational one of the following functional: 


$$
\begin{aligned}
F\left[\phi_{D^{0}}^{(m)}\right]= & \int_{0}^{\infty} J_{m}(r)\left\{\left[\frac{d}{d r} \phi_{D^{0}}^{(m)}(r)\right]^{2}-\frac{2}{r}\left[\phi_{D^{0}}^{(m)}(r)\right]^{2}\right. \\
& \left.+\left[E_{m}-E_{m}\left(D^{0}\right)\right]\left[\phi_{D^{0}}^{(m)}(r)\right]^{2}\right\} d r,
\end{aligned}
$$

where $E_{m}$ is defined by Eq. (6) and $J_{m}(r)$ is the radial part of the Jacobian volume element related to the free-electron density charge, $P_{m}(r)$, averaged over a sphere of radius $r$ centered at the ion location, and which is given by

$$
J_{m}(r)=4 \pi r^{2} P_{m}(r),
$$

with

$$
P_{m}(r)=\frac{1}{4 \pi} \int_{0}^{2 \pi} d \varphi \int_{0}^{\pi} d \theta \sin \theta f_{z}^{2}(r \cos \theta) R_{m}^{2}(r \sin \theta) .
$$

Calculating the derivative of the functional in Eq. (13), one can obtain a one-dimensional Euler-Lagrange equation for the function $\phi_{D^{0}}^{(m)}(r)$ :

$$
\begin{aligned}
& -\frac{1}{J_{m}(r)} \frac{d}{d r}\left[J_{m}(r) \frac{d}{d r} \phi_{D^{0}}^{(m)}(r)\right]-\frac{2}{r} \phi_{D^{0}}^{(m)}(r) \\
& \quad=\left[E_{m}\left(D^{0}\right)-E_{m}\right] \phi_{D^{0}}^{(m)}(r),
\end{aligned}
$$

which describes a hydrogenlike atom in a space with an effective fractional dimensionality. ${ }^{17,19}$ To obtain the corresponding $D^{0}$ energy levels, we solve this equation by using the trigonometric sweep method. ${ }^{23}$

The advantage of the procedure explained above lies in the fact that all information about any anisotropy of the system (confinement due to the heterostructure and/or magnetic field) is contained in the Jacobian $J_{m}(r)$, which is known analytically. This allows us, once the charge density distribution of the free electron in the heterostructure is known, to reduce significantly the computational time to solve the donor Hamiltonian.

We proceed in a similar way with the calculation of the ground and first excited state of the $D^{-}$by choosing a trial wave function with a well-defined $z$ component of the total magnetic number $L=m+m^{\prime}$ as follows:

$$
\begin{aligned}
\Psi_{D^{-}}^{\left(m m^{\prime}\right)}\left(\mathbf{r}_{1}, \mathbf{r}_{2}\right)= & \frac{1}{\sqrt{2}}\left[f_{m}\left(\mathbf{r}_{1}\right) f_{m^{\prime}}\left(\mathbf{r}_{2}\right) \pm f_{m^{\prime}}\left(\mathbf{r}_{1}\right) f_{m}\left(\mathbf{r}_{2}\right)\right] \\
& \times \phi_{D^{-}}^{\left(m m^{\prime}\right)}\left(\left|\mathbf{r}_{1}-\zeta\right|,\left|\mathbf{r}_{2}-\zeta\right|, r_{12}\right), \\
& \left(m, m^{\prime}=0, \pm 1\right),
\end{aligned}
$$

where the envelope function $\phi_{D^{-}}^{\left(m m^{\prime}\right)}$ takes into account the effect of the correlation between the electrons within the heterostructure produced by the Coulomb interaction. In this equation the sign $+(-)$ corresponds to the singlet (triplet) state, and $f_{m}\left(f_{m^{\prime}}\right)$ are the wave functions given by Eq. (6). Transferring the origin of the coordinate system to the ion position and following the procedure as described in Ref. 17 we can write the Schrödinger equation for the off-center $D^{-}$ system in a QD as a variational problem of the functional:

$$
\begin{aligned}
F\left[\phi_{D^{-}}^{\left(m m^{\prime}\right)}\right]= & \int d \mathbf{r}_{1} \int d \mathbf{r}_{2} J_{m m^{\prime}}\left(r_{1}, r_{2}\right)\left[\sum_{i=1}^{2}\left(\nabla_{i} \phi_{D^{-}}^{\left(m m^{\prime}\right)}\right)^{2}\right. \\
& +U\left(r_{1}, r_{2}, r_{12}\right)\left(\phi_{D^{-}}^{\left(m m^{\prime}\right)}\right)^{2}+\left(E_{m}+E_{m^{\prime}}-E\right) \\
& \left.\times\left(\phi_{D^{-}}^{\left(m m^{\prime}\right)}\right)^{2}\right],
\end{aligned}
$$

where

$$
\begin{aligned}
J_{m m^{\prime}}\left(r_{1}, r_{2}\right)= & \frac{1}{2}\left[P_{m m}\left(r_{1}\right) P_{m^{\prime} m^{\prime}}\left(r_{2}\right)\right. \\
& \left.+P_{m^{\prime} m^{\prime}}\left(r_{1}\right) P_{m m}\left(r_{2}\right) \pm 2 P_{m m^{\prime}}\left(r_{1}\right) P_{m^{\prime} m}\left(r_{2}\right)\right],
\end{aligned}
$$

$$
\begin{aligned}
P_{m m^{\prime}}(r)= & \frac{1}{4 \pi} \int_{0}^{2 \pi} d \varphi \int_{0}^{\pi} \sin \theta d \theta f_{z}^{2}(r \cos \theta+\zeta) \\
& \times R_{m}(r \sin \theta) R_{m^{\prime}}(r \sin \theta),
\end{aligned}
$$

and

$$
U\left(r_{1}, r_{2}, r_{12}\right)=-\frac{2}{r_{1}}-\frac{2}{r_{2}}+\frac{2}{r_{12}},
$$

where $r_{1}, r_{2}$ are the ion-electron separations for electron 1 and 2 , respectively, and $r_{12}$ is the distance between the two electrons, and $J_{m m^{\prime}}(r)$ is the Jacobian in an isotropic space with fractional dimension. In order to solve the functional (13) and calculate the $D^{-}$binding energy, we consider a simple three-parameter Hylleraas trial function for the envelope wave function as follows:

$$
\Phi_{D^{-}}\left(r_{1}, r_{1}, r_{12}\right)=\exp (-\alpha s)\left(1+\beta t^{2}+\eta u\right),
$$

where $\alpha, \beta$, and $\eta$ are variational parameters, and $s=r_{1}+r_{2}$, $t=r_{1}-r_{2}$, and $u=r_{12}$ are the Hylleraas coordinates. Following the Hylleraas procedure ${ }^{24}$ we obtain an explicit expression for the variational energy as a function of the parameters $\alpha$, $\beta$, and $\eta$ and we calculate the binding energy of the system by minimizing this expression with respect to those parameters:

$$
E\left(D^{-}\right)=\min _{\alpha, \beta, \eta} E\left(D^{-} ; \alpha, \beta, \eta\right)
$$

\section{COMPARISON BETWEEN THE TWO METHODS}

The binding energies for the $D^{0}$ and $D^{-}$ground and excited states are defined as

$$
\begin{gathered}
E_{b}\left(D^{0}\right)=E\left(W, \omega_{0}, B\right)-E_{T}\left(D^{0}\right), \\
E_{b}\left(D^{-}\right)=E\left(W, \omega_{0}, B\right)+E_{T}\left(D^{0}\right)-E_{T}\left(D^{-}\right),
\end{gathered}
$$

with $E\left(W, \omega_{0}, B\right)$ the energy of an electron in a quantum well with thickness $W$ and lateral parabolic confinement $\omega_{0}$ in the presence of an uniform magnetic field $B$ and $E_{T}(X)$ is the total energy of the complex $X$ in the presence of the quantum well and of the parabolic confinement and external magnetic 

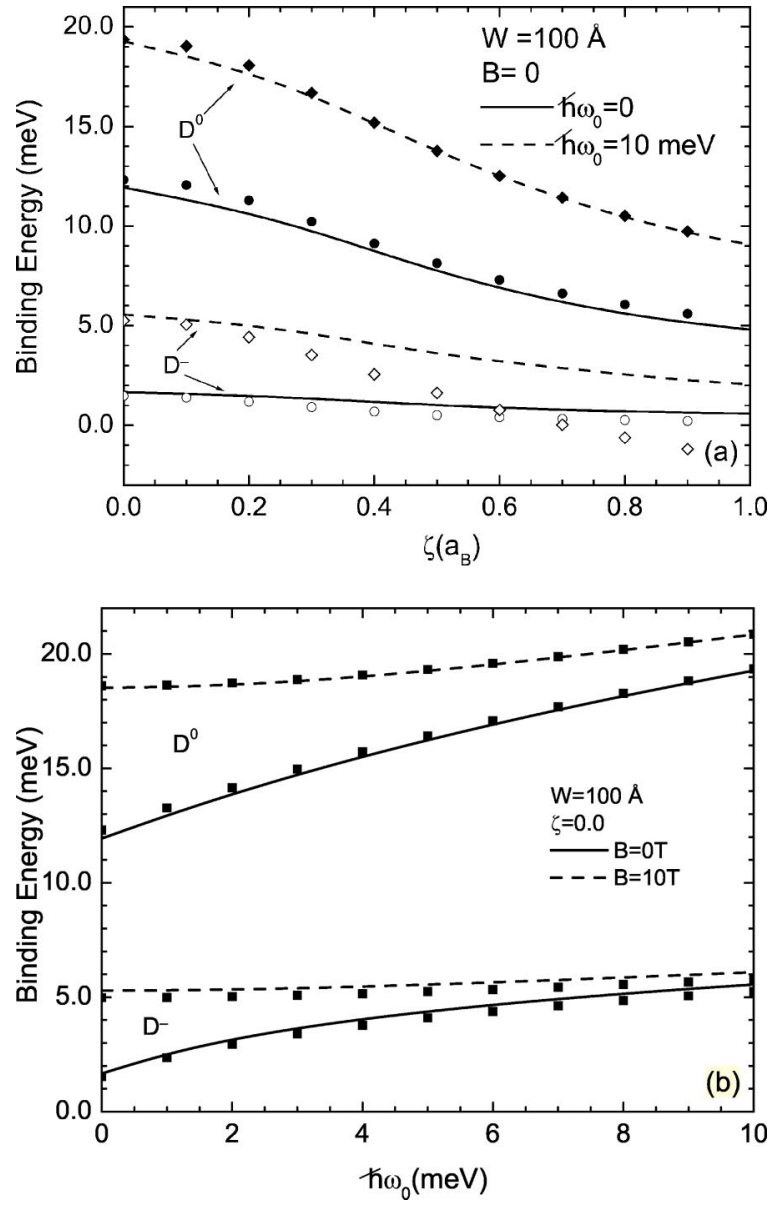

FIG. 1. Binding energies of the ground state of the donor and of the negatively charged donor obtained with the FDiff (symbols) and with the FDim (curves) methods. In (a) the binding energies are plotted vs the donor position for fixed values of the confinement potential. In (b) the binding energies are plotted vs the confinement potential energy for fixed values of the magnetic field and the donor is placed in the center of the dot, i.e., at $\zeta=0$.

field. For those systems the projection of the angular momentum along the $z$ direction is a good quantum number, due to the cylindrical symmetry of the confining structure. The groundstate of the negatively charged donor is the spin singlet with $L=0$ which is known to be the groundstate of the $D^{-}$in the absence of a magnetic field; the excited state is the first excited state at $B=0$ which has $L=-1$ and is a spintriplet state.

First we compare in Fig. 1 the results obtained with the FDiff (symbols) and the FDim (curves) in a QW for a GaAs well of width $W=100 \AA$. In Fig. 1(a) the binding energies are shown as a function of the position of the donor with respect to the center of the quantum well with (dashed curves) and without (full curves) confinement, while in Fig. 1(b) the binding energies of a center, $D^{0}$ and $D^{-}$, are shown as functions of the dot confinement for different external magnetic fields. We observe that the results obtained with both method for $B=0$ and different positions of the impurity are remarkably close, both for the $D^{0}$ and the $D^{-}$system. Notice also that both the $D^{-}$and the $D^{0}$ binding energies diminish with about $50 \%$ as the donor moves from the center of the well to about $0.75 a_{B}$ away from the center, see Fig. 1(a). Notice that for the $D^{0}$ the FDim binding energy is slightly lower than the FDiff one, while for $D^{-}$the FDim's are larger than the FDiff ones. This small discrepancy is not surprising, in fact the FDiff confines the electrons to a plane while the FDim allows them to move along the $z$ direction. This means that for $D^{0}$ the FDiff will slightly overestimate the attraction between the electron and the donor, especially when the donor is in the center of the well. For the negatively charged donor the electron-electron repulsion is also somehow artificially enhanced in the FDiff by the fact that both electrons are confined in a plane at the center of the well. The fact that the electrons are confined at the center of the well keeps them also further from the impurity when it is off-center, diminishing as a consequence the electron-donor attraction. The combination of this two effects is likely to lead to an underestimation of the binding energy by the FDiff method.

Next we consider the case when the donor is in the center of the well and the parabolic confinement is present, i.e., $\omega_{0} \neq 0$, Fig. 1(b), in the absence of a magnetic field. Notice that when $B=0$ the binding energy of both $D^{0}$ and $D^{-}$ground states are increased by the added confinement. While for the $D^{0}$ the binding energy increases slowly but constantly, for the $D^{-}$it tends to saturate. In fact in the $D^{0}$ the electron is forced closer to the donor by the extra confinement, which increases the binding energy. In the $D^{-}$the extra confinement, on the one hand, pushes the electrons closer to the donor, but on the other hand, it forces the electrons to be close to each other. Thus, there are two competing trends: the increase of the electron-donor attraction and the increase of the electron-electron repulsion, which almost balance each other. Similarly to the previous case the results from the two techniques are very close and the small discrepancy can be easily explained with the fact that the FDiff imposes a stronger confinement on the electrons in the $z$ direction with respect to the FDim technique.

If we apply a perpendicular magnetic field, e.g., $B=10 \mathrm{~T}$ in Fig. 1(b), the binding energy increases and the increase is larger for small confinement. We find that the results obtained for the binding energy of the ground state of both the $D^{0}$ and $D^{-}$systems with the two techniques are qualitatively similar, though the quantitative differences among the results for the $D^{-}$ground state is slightly increased. The quantitative difference can again be explained by the fact that different approximations are used in the two approaches. In particular, in this situation, the magnetic field pushes the electrons closer to each other and to the donor which is in the center of the well, and since in the FDiff they are not allowed to reaccommodate in the $z$ direction, the binding energy is then more strongly influenced than in the FDim, where the electrons can compensate for the extra confinement in the plane by reaccommodating in the $z$ direction.

Since the two techniques agree for the on-center $D^{0}$ and $D^{-}$with and without magnetic fields we want to see what happens if we have the off-center donor in a QD, i.e., $\omega_{0}$ $\neq 0$. In Fig. 1(a) we see that as the donor moves away from the center of the dots, the results obtained for the $D^{-}$with the two techniques diverge, while the results for the $D^{0}$ are still in very good agreement. The reason for the strong disagreement between the two techniques for the off-center $D^{-}$in a 

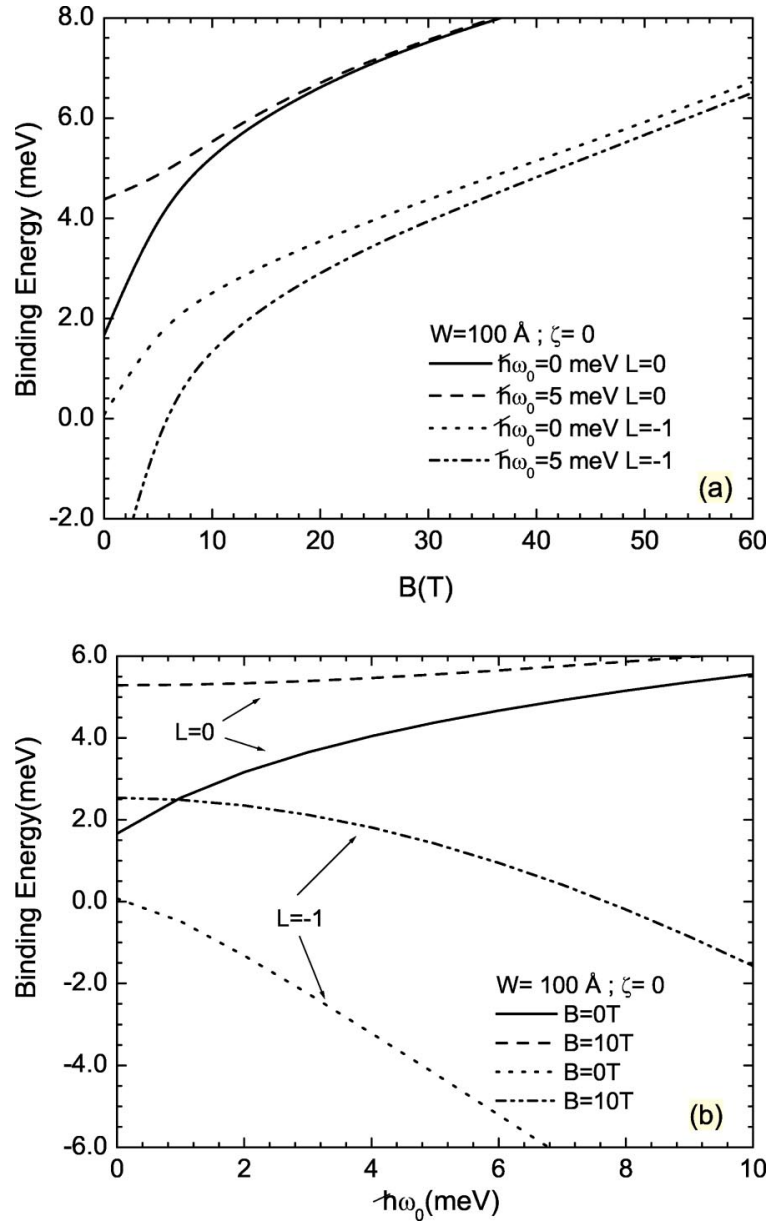

FIG. 2. Binding energies of the ground state and the first excited state of an on-center negatively charged donor. In (a) the binding energies are plotted vs the magnetic field for fixed values of the confinement potential. In (b) the binding energies are plotted vs the confinement potential energy for fixed values of the magnetic field.

QD is difficult to find. In fact while the small discrepancies found until now could easily be explained with the different approximations used, they are not enough to explain the large difference obtained if the donor is more than a quarter of the well width away from the center and, in fact, it signals the breakdown of the FDim approach. In particular, the electrons also in the FDim do not follow the donor that moves further than a quarter of the well width away from the center. Thus, the movement of the electron in the FDim approach cannot account for the large difference found between the two techniques.

\section{INFLUENCE OF THE POSITION OF THE DONOR ON THE ANGULAR MOMENTUM TRANSITIONS AND THE UNBINDING OF THE $D^{-}$}

We found that the FDiff and the FDim lead to similar results for the binding energies of the on-center $D^{0}$ and $D^{-}$ system. Since the FDim provides a faster calculation, we use this method to study the $D^{-}$on center system while we will use the more accurate FDiff for the off center $D^{-}$later in this paper. In Fig. 2 we present the binding energy of the ground and first excited state of the negatively charged donor versus the magnetic field applied in a QD with thickness $W$ $=100 \AA$ and confinement frequency $\hbar \omega_{0}=5 \mathrm{meV}$ and we compare them to the one obtained without in-plane confinement, i.e., $\hbar \omega_{0}=0$. The binding energy of the $L=0$ ground state of the $D^{-}$has a slow increase with the magnetic field in the low field region, while in a $\mathrm{QW}$ in this region the increase of the binding energy is quite fast with the magnetic field. However for large magnetic field, i.e., $B>20 \mathrm{~T}$, we find that the behavior of the negatively charged donor in the two nanostructures converges, i.e., becomes independent of the lateral confinement. The explanation for this is as follows: in a QW the energy of the state depends on $\gamma_{c} \approx B$ while for a quantum dot it depends on $\gamma=\sqrt{\gamma_{c}^{2}+\omega^{2}}$. This means that for $\gamma_{c} / \omega \leqslant 1$ the behavior of the $D^{-}$in the two nanostructures differs. In particular in the QD the dependence on the magnetic field is less strong. If $\gamma_{c} \gg \omega$ instead we have $\gamma \approx \gamma_{c}$ and consequently the two results converge, namely the confinement due to the magnetic field dominates the parabolic confinement. Notice that we have used here $\gamma_{c}$ and $\omega$ which are the $a$-dimensional correspondents of $B$ and $\omega_{0}$, because they give a better idea of the relative weight of the different terms contributing to the Hamiltonian.

For the first excited state $L=-1$ we observe that both in the QW and in the QD there is a fast increase of the binding energy of the state with the magnetic field. Notice that for $B=0$ the $L=-1$ state has zero binding energy in the absence of in-plane confinement and it is "unbound," i.e., positive Coulomb energy when the $D^{-}$system is confined. In this case in fact due to a projection of the angular momentum along the $z$ axis, which is nonzero, there is a linear dependence on $B$ both for the QD and the QW. Also in this case the behavior of the first excited state in the two nanostructures tends to converge for large magnetic fields. Notice that also in a QD when the donor is at the center of the well there is no magnetic-field-induced transition of the ground-state to a state with different $L$ [see Fig. 2(a)].

Next we studied the effects of a varying confinement potential, see Fig. 2(b). We observe that both in the presence and in the absence of an external magnetic field the energy of the ground state of the $D^{-}$increases with increasing confinement potential $\omega_{0}$, however we notice that the increase is slower when a magnetic field is applied. This result is somehow close to the one we reported in Fig. 2(a), for increasing $B$ and fixed $\omega_{0}$. On the other hand, the dependence of the first excited state on the confinement frequency is quite different from its dependence on the magnetic field $B$. We observe that with increasing parabolic confinement the binding energy of the excited state decreases and can even become negative, while in the case of increasing magnetic field the binding energy of the excited state increases.

The reason for this behavior is to be found in the fact that the parabolic confinement constrains the electrons around the position $\rho=0$, while the magnetic field action can be rewritten as the one of a displaced harmonic oscillator in which the electrons oscillate around the position $l_{0}$ that depends on the $z$ component of the angular momentum of the electron, i.e., $l_{0} \neq 0$. Thus, when a parabolic confinement is applied, the electrons are forced to stay closer and closer to each other, both in the ground and in the excited state. This potential 

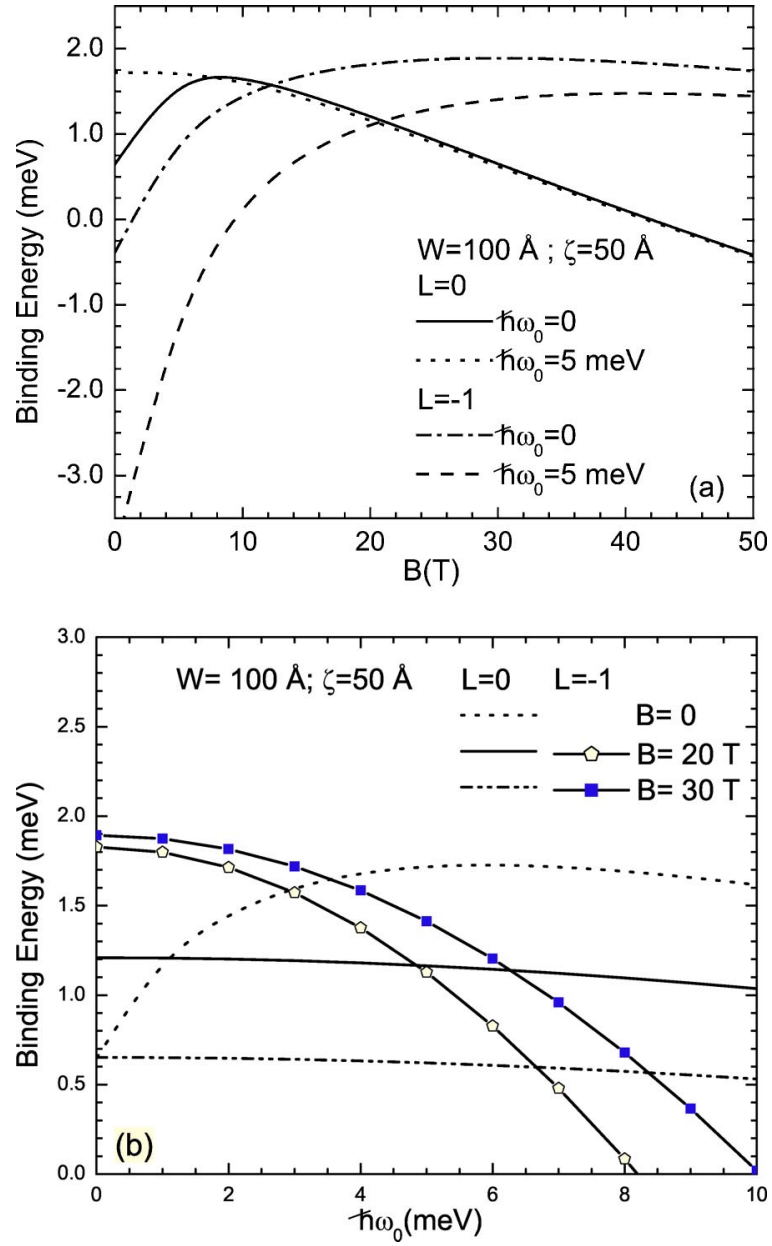

FIG. 3. Binding energies of the ground state and the first excited state of the negatively charged donor located at the well barrier interface. In (a) the binding energies are plotted vs the magnetic field for fixed values of the confinement potential. In (b) the binding energies are plotted vs the confinement potential energy for fixed values of the magnetic field.

adds extra positive energy which is larger with increasing $\omega_{0}$. When an external magnetic field is applied, the electrons in the excited state $L=-1$ are pushed further apart, which eases the repulsion between the electrons so that the binding energy increases, see Fig. 2(b).

Next we study the influence of the donor position on the binding energy of the ground and excited states. It is well known $^{12}$ that for negatively charged donors in quantum wells a displacement of the donor from the well center leads to angular momentum transitions of the ground state when applying an external uniform magnetic field. In a quantum dot such transitions can still be induced, however the presence of an additional parabolic confinement shifts the critical field to higher values and the transition energy to lower energies as compared to the $\omega_{0}=0$ case. In Figs. 3(a) and 3(b) we plot the binding energy for the ground state and the first excited state of a negatively charged donor in a quantum well of width $100 \AA$ when the donor is at the edge of the well. We compare those results with the results for a negatively charged donor in a quantum dot with the same thickness as the reference QW and a confinement potential of $5 \mathrm{meV}$ in the $x y$ plane. Notice that similarly to the case of the oncenter donor only the low magnetic field behavior of the binding energy for the ground state is influenced, i.e., it becomes less sensitive to a change in the magnetic field strength when we move from QW to QD, see Fig. 3(a). The binding energy of the excited state decreases when an extra confinement is present, but the qualitative behavior is not altered. The net effect of this is to shift the critical magnetic field, i.e., the field where the ground-state transition happens, to larger values and the transition energy to lower values.

The influence of the in-plane confinement potential on the $D^{-}$binding energy for a fixed magnetic field is shown in Fig. 3(b) for a donor positioned at the edge of the dot. Notice that without magnetic field the ground-state binding energy increases with $\omega_{0}$ up to about $0.3 \mathrm{R}_{y}$ and then for $\hbar \omega_{0}$ $\geqslant 6 \mathrm{meV}$ it starts to decrease. In the latter case the electrondonor attraction is decreased as compared to the on-center case. The donor and the electron are spatially separated along the $z$ axis and as a consequence the increase in the electronelectron repulsion due to the extra confinement diminishes the binding of the system. The excited state is not shown since it is unbound in this case.

When a magnetic field of, e.g., $20 \mathrm{~T}$ is applied we observe that as expected ${ }^{12}$ at $\omega_{0}=0$, the first excited state at $B=0$ becomes the ground state. However the energy of the excited state decreases with increasing confinement potential frequency and has a faster decrease than the $L=0$ ground-state energy. As a consequence for $\hbar \omega_{0} \geqslant 5.5 \mathrm{meV}$ the state with $L=0$ becomes again the ground state. Notice that the binding energy of the excited state decreases faster than the one of the ground state, which can be explained by the fact that the electrons in the excited state are farther apart from each other and are thus more sensitive to the extra localization.

The fact that in the presence of an in-plane confinement the critical magnetic field shifts to higher values and the energy decreases implies that a smaller number of angular momentum transitions occur before the $D^{-}$becomes unbound in the QD as compared to a similar situation in the QW case. In Fig. 4 we show the phase diagram for the case when the donor is a distance of $75 \AA$ away from the center of the dot in a $100-\AA$-wide $\mathrm{QW}$, i.e., the donor is located in the barrier. We observe that if $\omega_{0}=0$ there are up to five angular momentum transitions, but as the confinement is increased we notice that substantially smaller magnetic fields are required to unbind the negatively charged donor. Of course, in our dot system, unbinding means that the $D^{-}$bound state has a higher energy than the state consisting of a neutral donor and an extra electron. With other words, when adding a second electron to the donor system, the extra Coulomb energy is positive. The magnetic-field-induced unbinding of the $D^{-}$ system at high magnetic fields can be shown more clearly by resorting to the classical problem. As is well known in the limit of very high magnetic field the kinetic energy of the electrons freezes out and a classical problem with point particles results. Such a classical approach is given in the Appendix.

\section{CONCLUSION}

In conclusion we studied how the neutral and charged donors in a quantum well are influenced by an external para- 


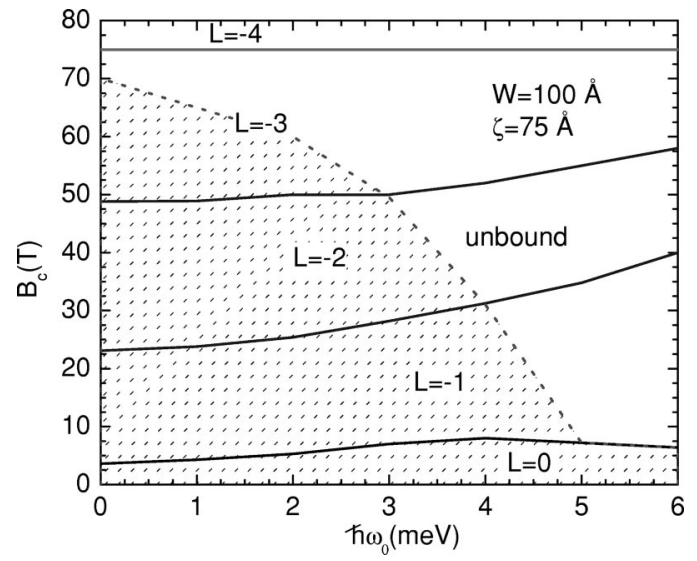

FIG. 4. Phase diagram for the ground state of a negatively charged donor confined into a quantum dot of thickness $100 \AA$ and lateral confinement $\omega_{0}$. The donor is positioned a vertical distance $75 \AA$ away from the center of the dot. $B_{c}$ indicates the critical transition field.

bolic confinement, i.e., transition from QW to QD. We also studied the influence of an external magnetic field. We found that the on-center $D^{0}$ and $D^{-}$ground-state binding energies are increased both by the extra confinement and by the external magnetic field. The $D^{-}$excited-state binding energy is, on the other hand, increased by an external magnetic field but decreased when a parabolic confinement is applied. For the off-center systems we found that the ground-state energy of the $D^{-}$is increased by the extra confinement. The effect of the external magnetic field and of the parabolic confinement on the ground-state of the $D^{-}$is similar, namely the binding energy is first increased up to a maximum after which it starts to decrease. The excited state of the $D^{-}$has a different dependence on the magnetic field and the confinement. While there is an increase with the magnetic field of the binding energy of the $L=-1$ state, at least at low field, the confinement only decreases the excited-state binding energy. In particular we found that the presence of an in-plane confinement shifts the magnetic field at which the angular momentum transitions occur to higher values, and as a consequence a smaller number of angular momentum transitions take place before the $D^{-}$becomes unbound in the QD as compared to the QW case.

\section{ACKNOWLEDGMENTS}

This work was supported by the FWO-Vl (Flemish Science Foundation), the Belgian Interuniversity Attraction Poles (IUAP), the Flemish Concerted Action (GOA) Programme, and the University of Antwerp (VIS). Clara Riva is supported by FWO-Vlaanderen. Ruthber Escorcia wishes to thank the Universidad del Magdalena for the permission to study at the University of Antwerp.

\section{APPENDIX : CLASSICAL APPROACH}

We present here a classical calculation that should coincide with the quantum results known for the $D^{-}$system in the very high magnetic field limit.

Let us first consider the $D^{-}$in a quantum well in the classical limit. The classical energy of two electrons which can be taken on a line in the presence of the impurity potential is

$$
E_{\text {class }}=-\frac{e^{2}}{\epsilon \sqrt{x_{1}^{2}+\zeta^{2}}}-\frac{e^{2}}{\epsilon \sqrt{x_{2}^{2}+\zeta^{2}}}+\frac{e^{2}}{\epsilon\left|x_{1}-x_{2}\right|},
$$

with $x_{1}>0$ and $x_{2}<0$ the classical coordinates of the two electrons, and $\zeta$ the distance between the impurity and the QW center. The configuration with the minimum energy is: $x_{1}=0$ and $x_{2}$ infinity, i.e., the symmetric state $x_{1}=-x_{2}$ is not the ground state. Which agrees with the findings ${ }^{12}$ that in high magnetic field the two-electron bound state evaporates.

Next we add a parabolic potential so that Eq. (A1) becomes

$$
E_{\text {class }}=\frac{m \omega_{0}}{2}\left(x_{1}^{2}+x_{2}^{2}\right)-\frac{e^{2}}{\epsilon \sqrt{x_{1}^{2}+\zeta^{2}}}-\frac{e^{2}}{\epsilon \sqrt{x_{2}^{2}+\zeta^{2}}}+\frac{e^{2}}{\epsilon\left|x_{1}-x_{2}\right|},
$$

and introducing dimensionless variables $\chi_{1}=x_{1} / \zeta$ and $\chi_{2}$ $=x_{2} / \zeta$ we obtain

$$
E_{\text {class }}=\frac{e^{2}}{\epsilon \zeta}\left[\alpha\left(\chi_{1}^{2}+\chi_{2}^{2}\right)-\frac{1}{\sqrt{\chi_{1}^{2}+1}}-\frac{1}{\sqrt{\chi_{2}^{2}+1}}+\frac{1}{\left|\chi_{1}-\chi_{2}\right|}\right],
$$

with $\alpha=\epsilon / e^{2} \times m \omega_{0} \zeta^{3} / 2$. Here the ground-state configuration is given by a single parameter $\alpha$. In particular we find that if $\alpha>0.025$, the state with minimum energy is symmetric, while for $\alpha<0.025$ the symmetric state is no longer the ground state and an asymmetric solution appears. An estimate for the confinement threshold can be easily done using the material parameters, e.g., we found that for a GaAs quantum well with the donor $75 \AA$ away from the center of the well, as we considered in the phase diagram presented in this work (see Fig. 4), the threshold confinement frequency is $6.3 \mathrm{meV}$. In this case the electrons are confined by the parabolic potential and consequently we cannot find a solution in which one of the electrons moves to infinity. However we find that the binding energy of the negatively charged donor is always negative, which agrees with the results found in this paper that for sufficiently high magnetic fields the $D^{-}$is no longer bound and this for any value of the confinement potential $\omega_{0}$. 
*Electronic address: clara.riva@ua.ac.be

†Electronic address: francois.peeters@ua.ac.be

${ }^{1}$ D. E. Phelps and K. K. Bajaj, Phys. Rev. B 27, 4883 (1983).

${ }^{2}$ C. J. Armistead, S. P. Najda, P. A. Makado, R. A . Stradling, P. C. Coulter, and G. E. Stillman, Solid State Commun. 48, 51 (1983).

${ }^{3}$ S. Holmes, J. P. Cheng, B. D. McCombe, and W. Schaff, Phys. Rev. Lett. 69, 2571 (1992).

${ }^{4}$ S. Huant, A. Mandray, J. Zhu, S. Louie, T. Pang, and B. Etienne, Phys. Rev. B 48, 2370 (1993); T. Pang and S. G. Louie, Phys. Rev. Lett. 65, 1635 (1990).

${ }^{5}$ E. R. Mueller, D. M. Larsen, J. Waldman, and W. B. Goodhue, Phys. Rev. Lett. 68, 2204 (1992).

${ }^{6}$ R. Chen, K. K. Bajaj, J. P. Cheng, and B. D. McCombe, Phys. Rev. B 51, 7714 (1995).

${ }^{7}$ J.-L. Zhu and S. Xu, Phys. Rev. B 50, 12175 (1994).

${ }^{8}$ J. M. Shi, F. M. Peeters, and J. T. Devreese, Phys. Rev. B 51, 7714 (1995); ibid. 57, 3900 (1998).

${ }^{9}$ J.-L. Zhu, D. L. Lin, and Y. Kawazoe, Phys. Rev. B 54, 16786 (1996).

${ }^{10}$ I. D. Mikhailov, F. J. Betancur, and J. H. Marin, Phys. Status Solidi B 220, 171 (2000).

${ }^{11}$ J.-L. Zhu, J.-H Zhao, W.-H. Duan, and B.-L. Gu, Phys. Rev. B 46, 7546 (1992).
${ }^{12}$ C. Riva, V. A. Schweigert, and F. M. Peeters, Phys. Rev. B 57, 15392 (1998).

${ }^{13}$ X-F. He, Phys. Rev. B 42, 11751 (1990); 43, 2063 (1991).

${ }^{14}$ Wenfang Xie, Physica B 315, 117 (2002).

${ }^{15}$ J. Z. García, P. Pietiläinen, and P. Hyvönen, Phys. Rev. B 66, 195324 (2002).

${ }^{16}$ F. H. Stillinger, J. Math. Phys. 18, 1224 (1977).

${ }^{17}$ P. Lefebvre, P. Christol, H. Mathieu, and S. Glutsch, Phys. Rev. B 52, 5756 (1995).

${ }^{18}$ E. Reyes-Gmez, A. Matos-Abiague, C. A. Perdomo-Leiva, M. de Dios-Leyva, and L. E. Oliveira, Phys. Rev. B 61, 13104 (2000); Physica B 302-303, 72 (2001).

${ }^{19}$ I. D. Mikaihlov, F. J. Betancur, R. A. Escorcia, and J. SierraOrtega, Phys. Status Solidi B 234, 590 (2002).

${ }^{20}$ I. D. Mikaihlov, F. J. Betancur, R. A. Escorcia, and J. SierraOrtega, Phys. Rev. B 67, 115317 (2003).

${ }^{21}$ F. M. Peeters and V. A. Schweigert, Phys. Rev. B 53, 1468 (1996).

${ }^{22}$ S. Flügge, Practical Quantum Mechanics (Springer-Verlag, New York, 1974).

${ }^{23}$ F. J. Betancur, I. D. Mikhailov, and L. E. Oliveira, J. Phys. D 31, 3391 (1998).

${ }^{24}$ H. A. Bethe and E. E. Salpeter, Quantum Mechanics of One- and Two-Electrons (Springer-Verlag, Berlin, 1957). 